\title{
Calorimetric study of block-copolymers of poly(n-butyl acrylate) and gradient poly( $n$-butyl acrylate-co-methyl methacrylate)
}

\author{
A.I. Buzin ，M. Pyda ，P. Costanzo , K. Matyjaszewski , B. Wunderlich
}

\begin{abstract}
The nanophase separation in diblock and triblock copolymers consisting of immiscible poly $(n$ butyl acrylate) (block A) and gradient copolymers of methyl methacrylate (MMA) and $n$ butyl acrylate ( $n \mathrm{BA}$ ) (block $\mathbf{M} / \mathbf{A})$ were investigated by means of their heat capacity, $C_{\mathrm{p}}$, as a function of the composition of the blocks $\mathbf{M} / \mathbf{A}$ and temperature. In all copolymers studied, both blocks are represented by their $C_{\mathrm{p}}$ and glass transition temperature, $T_{\mathrm{g}}$, as well as the broadening of the transition temperature range. The low temperature transition of the blocks $\mathbf{A}$ is always close to that of the pure poly ( $n$ butyl acrylate) and is independent of the analyzed compositions of the block copolymer, but broadened asymmetrically relative to the homopolymer due to the small phase size. The higher transition is related to the glass transition of the copolymer block of composition M/A. Besides the asymmetric broadening of the transition due to the phase separation, it decreases in $T_{\mathrm{g}}$ and broadens, in addition, symmetrically with increasing acrylate content. The concentration gradient is not able to introduce a further phase separation with a third glass transition inside the M/A block.
\end{abstract}

Keywords: Differential scanning calorimetry; Gradient copolymer; Block copolymer

\section{Introduction}

The blending of different polymers often results in improvement of properties exhibited by the individual components. Most polymers are incompatible, therefore, in blends, they do not dissolve. For thermodynamic reasons, the phases should separate on a macroscopic scale. Combining long sequences of the different polymers into block copolymers complicates this phase separation because the strong chemical bonds linking the different parts of a block copolymer must be located at the interface between the different phases. This strongly-bound interface causes the most change in the properties of the block copolymers when compared to the homopolymers of the same components. As a major result, the otherwise expected separation into macrophases of the chemically different, immiscible components is restricted in the block copolymer, and depending on the size of the blocks, microphase or even nanophase-separation is observed [1]. The need to minimize the surface free energy of the phase-separated samples develops a rich variety of periodic morphologies [2].

For linear copolymers, two extreme structures can be inferred: diblock copolymers, composed of two connected, incompatible polymer chains, and random copolymers, where different types of monomers are distributed along the chains. Besides these, more complicated distributions of the monomers were also studied, e.g. tapered-block copolymers [3 5], multiblock copolymers [6], gradient copolymers [7, $8]$, and alternating, copolyoligomers $[9,10]$. The gradient copolymers are actually intermediate between the aforementioned two extreme cases. They have a well-defined structure and composition which changes gradually from predominantly sequences of one comonomer to the other as a function of the copolymer chain length. Due to this composition distribution, the repulsive inter-chain interactions are smoothly changing along the chain. This structure is different from the case of simple block copolymers, where the repulsive interactions are confined to the junction of the blocks, and random copolymers, where the repulsive interactions are distributed along the chain. These atypical interactions are expected to result in unique thermal properties for the gradient copolymers. Up to date, 
however, only few and qualitative publications are available which report on thermal analysis of gradient copolymers $[11,12]$. In the present work we use conventional, quantitative DSC methods to study miscibility and phase separation based on heat capacity and glass transition temperatures for a series of diblock and triblock copolymers of poly(butyl acrylate) and gradient copolymers of butyl acrylate and methyl methacrylate with different compositions.

\section{Experimental}

The diblock and triblock copolymers studies are represented by the generalized formulae $\mathbf{A} \mathbf{M} / \mathbf{A}$ and A/M A $\mathbf{M} / \mathbf{A}$, respectively. The block $\mathbf{M} / \mathbf{A}$ is a copolymer of methyl methacrylate (MMA) and $n$-butyl acrylate ( $n \mathrm{BA})$, while the block A consists of pure poly(n-butyl acrylate) $(\mathrm{P} n \mathrm{BA})$. The copolymers were synthesized by atom transfer radical polymerization (ATRP) [13 15] starting from macroinitiators of $\mathrm{P} n \mathrm{BA}$ of well-defined lengths which are either monofunctional (for the A M/A diblock copolymers) or bifunctional (for the $\mathbf{A} / \mathbf{M} \quad \mathbf{A} \mathbf{M} / \mathbf{A}$ triblock copolymers). The block $\mathbf{M} / \mathbf{A}$ is not a statistical copolymer, but it is a gradient copolymer with higher MMA concentration than the average at the junction point with $\mathbf{A}$ and higher $n$ BA concentration than the average at the free ends. In the copolymerization stage of the synthesis, MMA is initially consumed at a faster rate, so that the residual $n \mathrm{BA}$ concentration increases and leads to the higher $n \mathrm{BA}$ concentration towards the chain ends, thus, forming a natural gradient block.

\subsection{Synthesis of macroinitiator}

A flask was loaded with $\mathrm{CuBr}, 1,4$-dimethoxybenzene and a stir bar, which was then vacuum/backfilled with $\mathrm{N}_{2}$ (three times). Next, purged $n$-butyl acrylate ( $n \mathrm{BA})$ and $N, N, N^{\prime}, N^{\prime \prime}, N^{\prime \prime}$-pentamethyldiethyltriamine (PMDETA) were added, via $\mathrm{N}_{2}$ purged syringes. The solution was stirred until homogeneous, and the appropriate initiator was added (methyl-2-bromopropionate monofunctional; dimethyl2,6-dibromoheptadionate difunctional) via a purged syringe. An initial sample was taken, and the flask was placed in an $353 \mathrm{~K}$ oil bath for $240 \mathrm{~min}$. Three samples were dissolved in $\mathrm{CDCl}_{3}$, acetone, and THF for NMR, GC, and GPC analysis, respectively. The remaining sample was dissolved in acetone and passed through a column of alumina, excess solvent was removed followed by precipitation in 50:50 $\mathrm{H}_{2} \mathrm{O} / \mathrm{MeOH}$, and finally dried under vacuum.

\subsection{Synthesis of gradient copolymers}

A flask was loaded with $n$ BA macroinitiator and a stirrer bar, which was put under vacuum for at least $30 \mathrm{~min}$. Purged anisole was added to dissolve the polymer. A second flask was loaded with $\mathrm{CuCl}$, bis(2-pyridylmethyl)-octadecylamine (BPMODA) and a stirrer bar, which was then vacuum/backfilled with $\mathrm{N}_{2}$ (three times). Purged MMA and $n$ BA were added to the second flask in accordance with the desired characteristics of the gradient segment. The solution was stirred until homogeneous, and then transferred by syringe to the first flask, which was then placed into an $353 \mathrm{~K}$ oil bath for $24 \mathrm{~h}$. Three samples were dissolved in $\mathrm{CDCl}_{3}$, acetone, and THF for NMR, GC, and GPC analysis, respectively. The remaining sample was dissolved in acetone and passed through a column of alumina, excess solvent was removed followed by precipitation in 50:50 $\mathrm{H}_{2} \mathrm{O} / \mathrm{MeOH}$, and finally dried under vacuum. The structural characterization of the copolymers used are shown in Table 1. The composition is known from synthesis and quantitative NMR. The molar masses were measured by exclusion chromatography in THF $\left(1 \mathrm{~g} \mathrm{l}^{1}\right)$ calibrated with polystyrene. The data were rounded to two digits. Homopolymers for comparative study were poly( $n$-butyl acrylate) (Aldrich, $M_{\mathrm{w}} \quad 99,000 \mathrm{Da}$ ) and poly(methyl methacrylate) (Scientific Polymer Products, Inc., $M_{\mathrm{w}} \quad 35,000 \mathrm{Da}$ ).

\subsection{Calorimetric characterization}

The DSC study was carried out using a powercompensated Perkin Elmer DSC7, known to yield heat capacities with a precision of about $\pm 1 \%$ [16]. Dry $\mathrm{N}_{2}$ gas with a flow rate of $20 \mathrm{~cm}^{3}$ min ${ }^{1}$ was purged through the DSC cell. Cooling was accomplished using a mechanical refrigerator (IntraCooler). Typical sample masses for the standard DSC experiments were $1020 \mathrm{mg}$. The samples were weighed on a Cahn-28 automatic electro-balance with an accuracy of $\pm 0.001 \mathrm{mg}$.

The as-prepared samples were first heated to $423 \mathrm{~K}$ and kept at this temperature for $5 \mathrm{~min}$ to erase any prior thermal history. Then the samples were cooled to $205 \mathrm{~K}$ to set the structure to be analyzed by subsequent heating to $423 \mathrm{~K}$. The heating and cooling rates were $10 \mathrm{~K} \mathrm{~min}{ }^{1}$. The heat-flow rate was initially calibrated with the heat of fusion of indium $\left(28.45 \mathrm{~J} \mathrm{~g}^{1}\right)$. The heat capacity obtained was then refined by correction with a reference run of sapphire over the same temperature range as the sample [17]. The calorimeter asymmetry between the empty reference and sample calorimeters was eliminated with an empty-pan run used as a baseline for the heat-flow rate of the sample and calibration runs.

The glass transition temperature was chosen at the $50 \%$ change of the heat capacity which is close to the point of inflection. Also observed were the extrapolated temperatures of the beginning $\left(T_{1}\right)$ and end of the glass transition $\left(T_{2}\right)$, taken at the intersection of the tangent at the point of inflection with the extrapolated heat capacities of the solid and liquid phases, respectively. These temperature evaluations were made based on the experimental data, before comparison with data from the ATHAS data bank, as will be discussed below. The difference $T_{2} \quad T_{1}$ is a measure for 


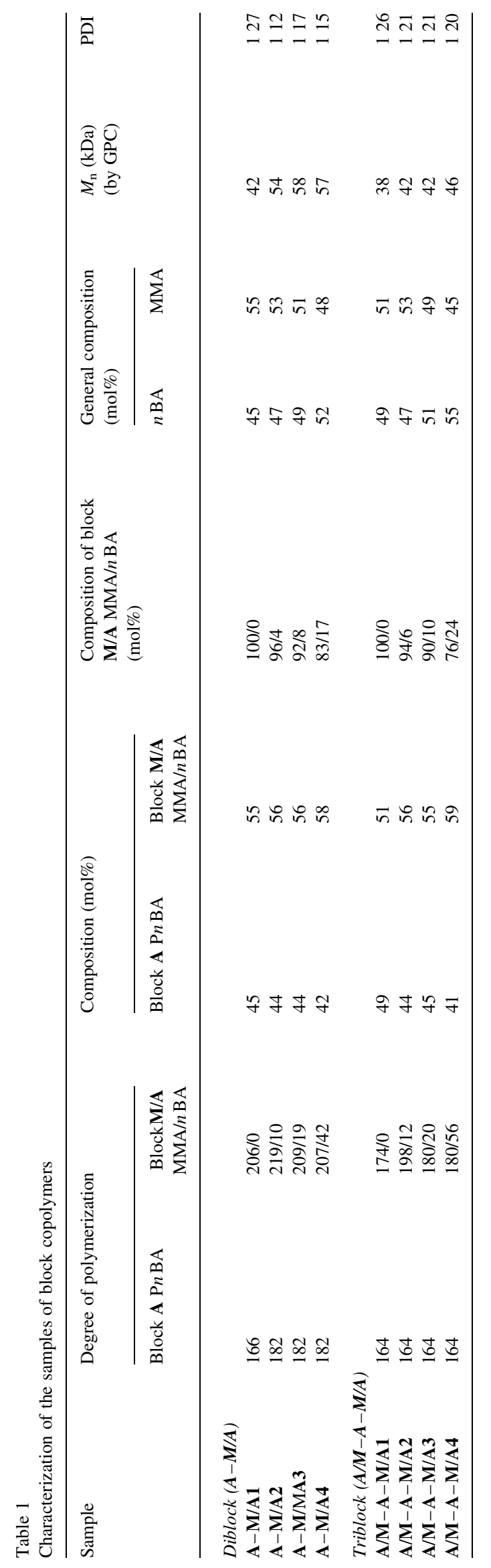

the breadth of the glass transition. The asymmetry of the glass transitions was characterized, in addition, whenever possible, by finding the temperatures $T_{\mathrm{b}}$ and $T_{\mathrm{e}}$, which were taken as the temperatures below and above the glass transition where the measured heat capacity begins to deviate from a linear the baseline of the glassy phase, and attains the linear baseline of the liquid phase, respectively. From the good fit to the data bank data on the homopolymers [18], one can estimate that the precision in heat capacity reached that common for the used instrumentation $( \pm 1 \%)$. Multiple measurements (3 5) were made on all samples.

\section{Results}

In Fig. 1(a) (d), the heat capacities are shown for the four A M/A-copolymers, measured after cooling from the melt. In the figures, the squares $(\square)$ represent the averaged data-bank heat capacities of the homopolymers of the components calculated from the recommended heat capacity tables available in the ATHAS Data Bank [18]. In the region between glass transitions of $\mathrm{P} n \mathrm{BA}$ and PMMA all $\mathrm{P} n \mathrm{BA}$ repeating units were taken to be in the liquid state, whereas all PMMA repeating units were taken to be solid. The crosses (+) in Fig. 1(b) (d) between the two glass transitions were calculated for the case that only the part of $\mathrm{P} n \mathrm{BA}$ contained in the block $\mathbf{A}$ is in the liquid state, whereas in the gradient section, both the $\mathrm{P} n \mathrm{BA}$ and the PMMA repeating units in the block $\mathbf{M} / \mathbf{A}$ are solid, a case more likely, at least on the low-temperature side of the region between the glass transitions. The dashed and dotted lines of reference represent the sums of the heat capacities of the proper fractions of homopolymers in the solid and liquid states, respectively, excluding the transition effects by extrapolation into the temperature range of interest. These lines, together with the continuous recordings of the DSC experiment, were used as baselines for the calculation of the glass transition parameters which are listed in Table 2.

At temperatures below the glass transition of $\mathrm{P} n \mathrm{BA}$, when both components are in the solid state, and in the region above $T_{\mathrm{g}}$ of PMMA, when both components are in the liquid state, the heat capacities of the $\mathbf{A} \mathbf{M} / \mathbf{A}$ copolymers exhibit fully additive behavior. The experimental heat capacity data for the region of temperatures below $T_{\mathrm{g}}$ of $\mathrm{P} n \mathrm{BA}$ are not completed in the figures, because the temperature range of the calorimeter is limited when using the IntraCooler as a cooling accessory. Heat capacities of all copolymers, however, were also investigated at lower temperatures (with lower precision) using liquid nitrogen cooling (see Fig. 1(a)). It has been found that ultimately the heat capacities are in good agreement with the baselines drawn from the ATHAS Data Bank using the assumption of additivity.

Both P $n$ BA and PMMA are non-crystallizable, amorphous polymers, and all their block copolymers demonstrate 

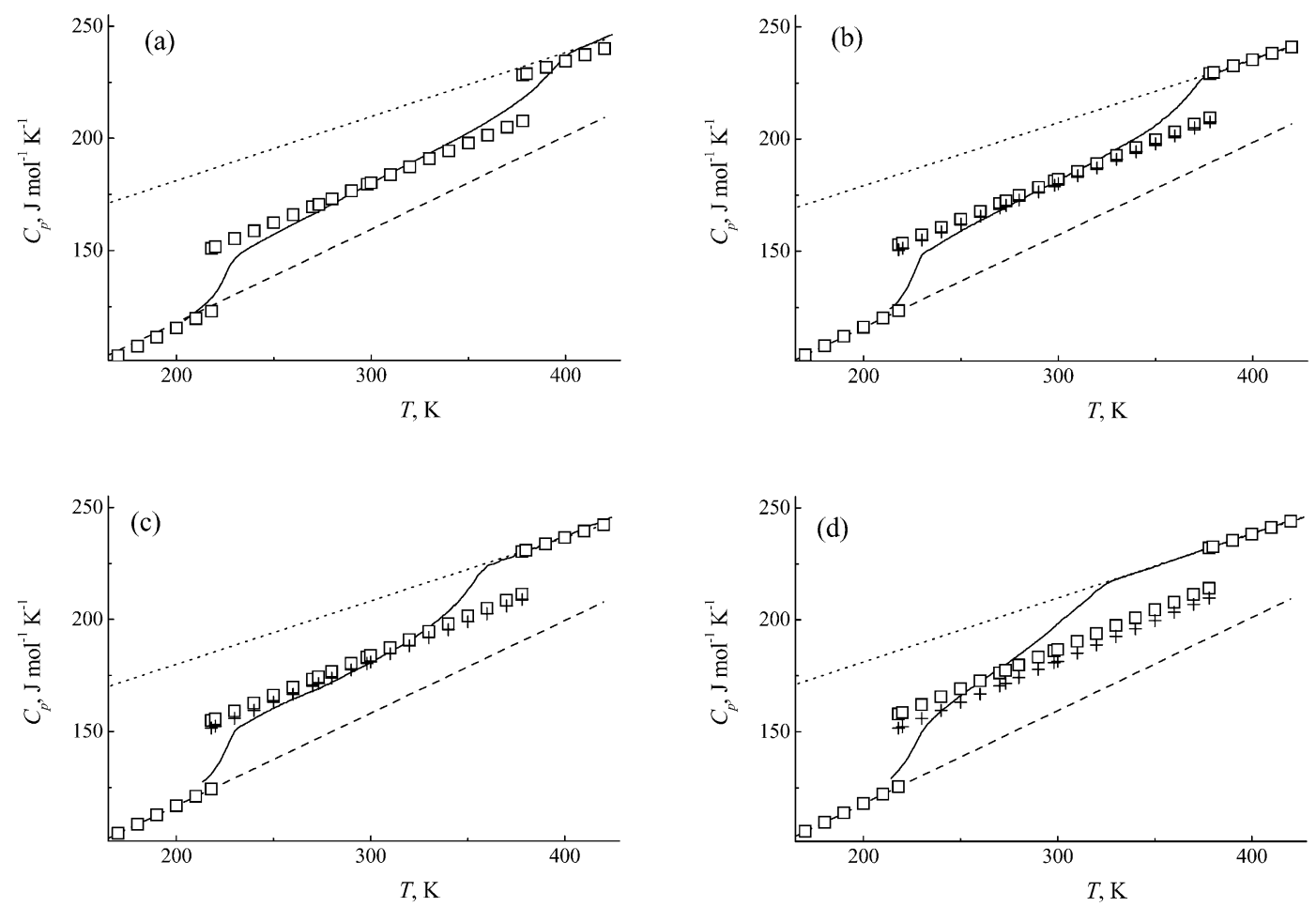

Fig. 1. Heat capacities of the $\mathbf{A} \mathbf{M} / \mathbf{A}$ copolymers as a function of temperature. Parts (a) (d) display the data on $\mathbf{A}$ M/A1, A M/A2, A M/A3, and A $\mathbf{M} / \mathbf{A 4}$, respectively, as shown in Table 1. For transition parameters see Table 2.

two glass transition temperatures. The parameters of the two glass-transitions are summarized in Table 2 and compared to the also measured transitions of the homopolymers. All low-temperature glass transition temperatures of the eight A $\mathbf{M} / \mathbf{A}$ and $\mathbf{A} / \mathbf{M}$ A $\mathbf{M} / \mathbf{A}$ copolymers are very similar at $225 \pm 2.5 \mathrm{~K}$ and agree with the newly measured $\mathrm{P} n \mathrm{BA}$. They somewhat exceed the $T_{\mathrm{g}}$ of $\mathrm{P} n \mathrm{BA}$ in the ATHAS Data Bank, which is $218 \mathrm{~K}$ [18]. The jump in the heat capacity in the region of the first $T_{\mathrm{g}}$ of the copolymers is not directly comparable because of different content of $n \mathrm{BA}$ in the blocks in the copolymer. The value of the first $\Delta C_{\mathrm{p}}$ per mole $x_{1}$ of $n \mathrm{BA}$ from the block $\mathbf{M} / \mathbf{A}$, however, is only $49 \pm 4 \mathrm{~J} \mathrm{~mol}^{1} \mathrm{~K}^{1}$ for the $\mathbf{A} \mathbf{M} / \mathbf{A}$-copolymers and $51 \pm 6 \mathrm{~J} \mathrm{~mol}^{1} \mathrm{~K}^{1}$ for all eight copolymers. This value is lower than the $\Delta C_{\mathrm{p}}$ at the glass transition for pure $\mathrm{P} n \mathrm{BA}$, which is $61.4 \mathrm{~J} \mathrm{~mol}^{1} \mathrm{~K}^{1}$ according to Table 2, and $62.3 \mathrm{~J} \mathrm{~mol}{ }^{1} \mathrm{~K}^{1}$ according to the literature [18]. The reason for the low value is the asymmetric broadening of the glass transition due to the size effect, to be described below.

The second glass transition temperature in the $\mathbf{A}$ M/A1

Table 2

Parameters of the glass transitions in the investigated homopolymers and their diblock and triblock copolymers

\begin{tabular}{|c|c|c|c|c|c|c|c|c|c|c|c|c|c|c|}
\hline \multirow[t]{2}{*}{ Sample } & \multicolumn{7}{|c|}{ Block A } & \multicolumn{7}{|c|}{ Block $\mathbf{M} / \mathbf{A}$} \\
\hline & $\begin{array}{l}T_{\mathrm{g}} \\
(\mathrm{K})\end{array}$ & $\begin{array}{l}\Delta C_{\mathrm{p}} \\
\left(\begin{array}{lll}\mathrm{J} \text { mol } & { }^{1} \mathrm{~K}^{1}\end{array}\right)\end{array}$ & $\begin{array}{l}\Delta \mathrm{C}_{\mathrm{p}} / x_{1} \\
\left(\begin{array}{lll}\mathrm{J} \text { mol } & { }^{1} \mathrm{~K} & \\
\end{array}\right)\end{array}$ & $\begin{array}{l}T_{1} \\
(\mathrm{~K})\end{array}$ & $\begin{array}{l}T_{2} \\
(\mathrm{~K})\end{array}$ & $\begin{array}{l}T_{\mathrm{e}} \\
(\mathrm{K})\end{array}$ & $\begin{array}{l}T_{2}-T_{1} \\
(\mathrm{~K})\end{array}$ & $\begin{array}{l}T_{\mathrm{g}} \\
(\mathrm{K})\end{array}$ & $\begin{array}{l}\Delta C_{\mathrm{p}} \\
\left(\begin{array}{lll}\mathrm{J} \text { mol } & 1 & \\
& & \end{array}\right)\end{array}$ & $\begin{array}{l}T_{\mathrm{b}} \\
(\mathrm{K})\end{array}$ & $\begin{array}{l}T_{1} \\
(\mathrm{~K})\end{array}$ & $\begin{array}{l}T_{2} \\
(\mathrm{~K})\end{array}$ & $\begin{array}{l}T_{\mathrm{e}} \\
(\mathrm{K})\end{array}$ & $\begin{array}{l}T_{2}-T_{1} \\
(\mathrm{~K})\end{array}$ \\
\hline \multicolumn{15}{|l|}{ Homopolymers } \\
\hline $\begin{array}{l}\text { P } n \text { BA } \\
\text { PMMA }\end{array}$ & 223 & 61.4 & 61.4 & 217 & 229 & 236 & 12 & 383 & 36.3 & 316 & 375 & 390 & 390 & 15 \\
\hline \multicolumn{15}{|l|}{ Diblock $(\boldsymbol{A} \quad \boldsymbol{M} / \boldsymbol{A})$} \\
\hline A $\mathbf{M} / \mathbf{A} 1$ & 224 & 20.1 & 44.7 & 218 & 230 & 240 & 12 & 388 & 15.6 & 355 & 375 & 402 & 403 & 28 \\
\hline A $\mathbf{M} / \mathbf{A} 2$ & 224 & 21.2 & 48.2 & 218 & 231 & 246 & 13 & 365 & 13.8 & 335 & 354 & 375 & 376 & 21 \\
\hline A $\mathbf{M} / \mathbf{A} \mathbf{3}$ & 223 & 22.5 & 51.1 & 215 & 231 & 243 & 16 & 347 & 21.2 & 301 & 333 & 361 & 362 & 28 \\
\hline A $\mathbf{M} / \mathbf{A} 4$ & 224 & 22.8 & 54.3 & 214 & 235 & 248 & 21 & 305 & 12.4 & 275 & 284 & 325 & 327 & 41 \\
\hline \multicolumn{15}{|c|}{ Triblock $(\boldsymbol{A} / \boldsymbol{M} \quad \boldsymbol{A} \quad \boldsymbol{M} / \boldsymbol{A})$} \\
\hline $\mathbf{A} / \mathbf{M} \quad \mathbf{A} \quad \mathbf{M} / \mathbf{A} 1$ & 222 & 28.1 & 57.3 & 212 & 231 & 247 & 18 & 374 & 13.4 & 339 & 356 & 392 & 395 & 36 \\
\hline $\mathbf{A} / \mathbf{M} \quad \mathbf{A} \quad \mathbf{M} / \mathbf{A} 2$ & 225 & 23.1 & 52.5 & 215 & 236 & 249 & 21 & 358 & 10.6 & 332 & 344 & 371 & 375 & 27 \\
\hline $\mathbf{A} / \mathbf{M} \quad \mathbf{A} \quad \mathbf{M} / \mathbf{A} \mathbf{3}$ & 228 & 23.4 & 52.0 & 213 & 242 & 254 & 29 & 335 & 10.0 & 302 & 317 & 352 & 354 & 35 \\
\hline $\mathbf{A} / \mathbf{M} \quad \mathbf{A} \quad \mathbf{M} / \mathbf{A} 4$ & 229 & 18.0 & 43.9 & 209 & 248 & 254 & 39 & & & & & 319 & & \\
\hline
\end{tabular}



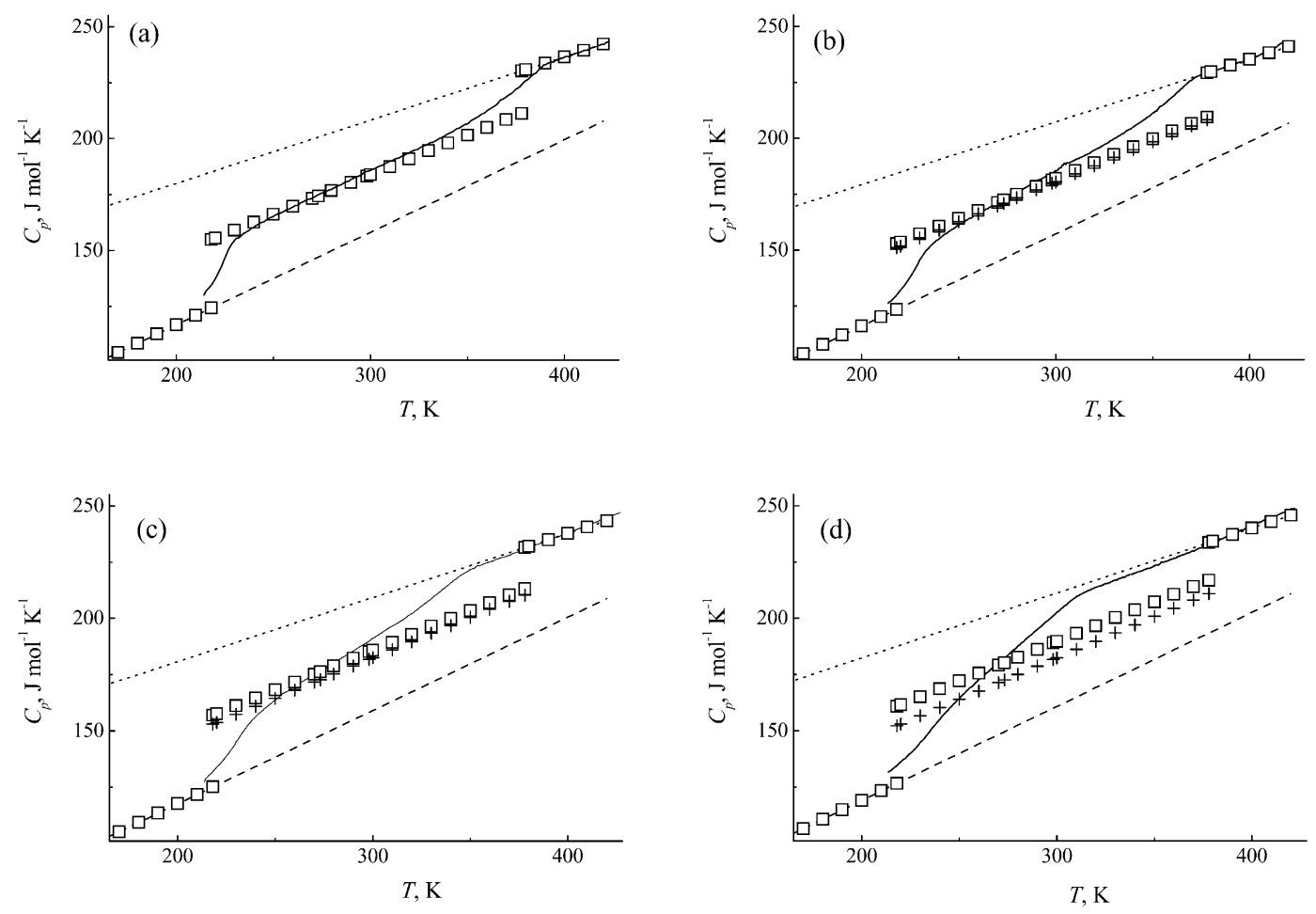

Fig. 2. Heat capacity of the $\mathbf{A} / \mathbf{M}$ A $\mathbf{M} / \mathbf{A}$ copolymers as a function of temperature. Parts (a) (d) display the data on $\mathbf{A} / \mathbf{M}$ A $\mathbf{M} / \mathbf{A 1}, \mathbf{A} / \mathbf{M}$ A $\mathbf{M} / \mathbf{A 2}, \mathbf{A} / \mathbf{M}$ A $\mathbf{M} / \mathbf{A 3}$, and $\mathbf{A} / \mathbf{M}$ A $\mathbf{M} / \mathbf{A 4}$, respectively, as shown the Table 1. For transition parameters see Table 2.

and A/M A A/M1 samples at 388 and $374 \mathrm{~K}$ are not far from the $T_{\mathrm{g}}$ of pure PMMA of $383 \mathrm{~K}$ as one would expect for pure PMMA blocks. The ATHAS Data Bank lists $378 \mathrm{~K}$ [18] for the $T_{\mathrm{g}}$ of PMMA. Again, as will be shown below, the asymmetry of the glass transition may introduce the somewhat larger spread of the PMMA glass transitions. In the other three A M/A samples, the second $T_{\mathrm{g}}$ is much lower than the $T_{\mathrm{g}}$ of PMMA. With increase in the content of $n \mathrm{BA}$ in the $\mathbf{M} / \mathbf{A}$ block, it shifts to the region of lower temperatures, towards the $T_{\mathrm{g}}$ of $\mathrm{P} n \mathrm{BA}$. In addition, the second $T_{\mathrm{g}}$ broadens with an increase in content of $n \mathrm{BA}$ in the M/A block, as seen when inspecting Fig. 1(b) (d).

The heat capacity plots for the four $\mathbf{A} / \mathbf{M} \mathbf{A}$ M/Acopolymers are shown in Fig. 2(a) (d). The calculated lines are generated as described for Fig. 1. The behavior of this series of copolymers is similar to that of the $\mathbf{A} \mathbf{M} / \mathbf{A}$ copolymers. Their heat capacities are additive, both, in the region of temperatures below the glass transition of $\mathrm{P} n \mathrm{BA}$ where both components are in the solid state, and in the region above $T_{\mathrm{g}}$ of PMMA, where both components are in the liquid state. All the $\mathbf{A} / \mathbf{M} \mathbf{A} \mathbf{M} / \mathbf{A}$-copolymers also demonstrate two glass transition temperatures, as listed in Table 2. All the first glass transitions of the copolymers are similar to the A M/A copolymers, as pointed out in the description of Fig. 1.

The second glass transition behaves also similar to the A M/A-copolymers. Its temperature decreases and the region of glass transition broadens with increase in the $n \mathrm{BA}$-content in the block $\mathbf{M} / \mathbf{A}$. The glass transition region in the $\mathbf{A} / \mathbf{M} \mathbf{A} \mathbf{M} / \mathbf{A} 4$ copolymer is so broad, that it is hard to distinguish the two transitions. From the discussion of the heat capacity plot, however, it becomes clear that this copolymer still has two glass transitions, i.e. it remains incompatible over the full range of concentration.

\section{Discussion}

There are two major effects which can influence the glass transitions in block copolymers: (A) the sizes of the phases, and (B) the solubility of the components in each other. If both blocks of the copolymer are incompatible, the segment lengths determine the sizes of the phases as microphase or nanophase and the separating phases will have a morphology that depends on their size-ratio [2].

The glass transition temperatures, $T_{\mathrm{g}}$, of both components are affected by the continuing molecules that cross the interface. If the second phase is less mobile, the end of the glass transition stretches to higher temperature as one approaches the interface. If, on the other hand, the second phase is more mobile, the beginning of the glass transition at the interface is broadened to lower temperature. When analyzing the two glass transitions of a block copolymer, one finds, thus, that the beginning of the overall glass transition region at the lower temperature and the end of glass transition at higher temperature will be constant, but the two midpoints of the transitions, the glass transition temperatures move towards each other because of the 
asymmetric broadening of the transition. This effect should be seen by the changes in $T_{\mathrm{b}}$ and $T_{\mathrm{e}}$. If the phase size is in the micrometer range or larger, this asymmetric broadening of the glass transition region is small because of the negligible specific interface area, as was shown by an earlier, quantitative analysis of the styrene/ $\alpha$-methylstyrene block copolymer system [19].

In the present case, however, the $\mathrm{P} n \mathrm{BA}$ sections which define the interface for both types of the analyzed block copolymers have an average of only 171 repeating units, as can be seen from Table 1. This length corresponds to an extended-chain length of approximately $45 \mathrm{~nm}$. The RMS end-to-end chain length of a random coil of this length in the melt or glass is about $10 \mathrm{~nm}$, a value calculated by assuming the typical expansion coefficient of the freely-jointed, random-flight, mean-square end-to-end distance for an acrylate to its real dimensions to be about 7 10. The molar fraction of the $\mathrm{P} n \mathrm{BA}$ segments is not far from 0.5 for all samples, which results in a lamellar morphology [2] which allows to quantify the interface. Only a small amount of ordering is expected due to the location of the P $n \mathrm{BA}$ segment-ends at the interface, i.e. the lamellar phases approach a nanophase thickness with a specific surface area of as much as $200 \mathrm{~m}^{2} \mathrm{~g}{ }^{1}$ when assuming the RMS end-toend length of $10 \mathrm{~nm}$ is a measure of the lamellar thickness. Such a large surface-to-volume ratio can lead to a substantial change in the breadth of the glass transition [19].

If partial solubility exists for one component in the other, or if both components are partially soluble in each other, either one or both of the glass transition temperatures, $T_{\mathrm{g}}$, will move towards the other, respectively. In addition the corresponding $\Delta C_{\mathrm{p}}$ must change according to the solubility. Furthermore, in the dissolution of longer sequences of identical repeating units, the glass transition region broadens symmetrically about $T_{\mathrm{g}}$. This is not the case in random copolymers where the glass transition also changes, but the breadth of the glass transition does not exceed that of the homopolymers, as was shown for example for increasingly brominated poly(2,6-dimethyl-1,4-phenylene oxide) [20]. For complete solubility of both components in block copolymers, as well as for blends of homopolymers, there is only one, broad glass transition and the beginning of the lower and the end of the upper glass transition move closer to each other, but never reaches the narrow glass transition range of a random copolymer, as was also documented on the styrene/ $\alpha$-methylstyrene system of homopolymers of varying molar mass by DSC [21].

The two glass transitions in the DSC traces of the samples investigated in this paper which consist of blocks of pure $\mathrm{P} n \mathrm{BA}$ and PMMA of approximately equal contents (A M/A1 and A/M A M/A1) show reasonable agreement with the glass transition temperatures of the homopolymers, suggesting that there is little miscibility between the components. The constancy of $T_{1}$ for block $\mathbf{A}$ of all nine homo- and copolymers, and $T_{2}$ and $T_{\mathrm{e}}$ for the PMMA and the $\mathbf{M} / \mathbf{A} 1$ and $\mathbf{A} / \mathbf{M} \quad \mathbf{A} \quad \mathbf{M} / \mathbf{A 1}$ copolymer proves similarly pure $\mathrm{P} n \mathrm{BA}$ and PMMA phases, at least within the interior of the phases. The Figs. 1(a) and 2(a), however, indicate a strong asymmetry between the glass transitions, seen best by the crossing of the measured and calculated heat capacities at about $300 \mathrm{~K}$. For truly identical behavior, the measured $C_{\mathrm{p}}$ should follow the calculated $C_{\mathrm{p}}$, marked by $\square$ in the temperature region between the glass transitions. This change in slope of the measured heat capacity leads to the noticeable decrease in $\Delta C_{\mathrm{p}} / x_{1}$ in Table 2 and masks the changes in $T_{\mathrm{e}}$ of $\mathbf{A}$ and $T_{\mathrm{b}}$ of $\mathbf{M} / \mathbf{A}$. Comparing these data to the similar analysis in the literature of a poly(styrene-block$\alpha$-methyl styrene) this should be caused by the smallness of the phases [19]. The mobile A-phase is bound to the glassy M/A-phase, i.e. the outer layers of $n$ BA have their mobility reduced and a part of the glass transition moves to higher temperature. The opposite effect is observed for the M/Aphase where the surface attachment to the liquid A-phase lowers the glass transition, not because of solubility, but because of molecular mobility of the surface layer of the PMMA.

Additional changes in the glass-transition behavior are observed in the other three $\mathbf{A} \mathbf{M} / \mathbf{A}$ samples shown in Fig. 1. They also exhibit two glass transitions. The lowtemperature $T_{\mathrm{g}}$ is, again, in reasonable agreement with $T_{\mathrm{g}}$ of $\mathrm{P} n \mathrm{BA}$. This allows to suggest that the first $T_{\mathrm{g}}$ belongs to the blocks A with little change from A M/A1 to A M/A4. In these copolymers the blocks of $160 \quad 180 n$ BA units are still phase-separated and only influenced by the rigidity of the second block due to the smallness of the phase. The value of $T_{2} \quad T_{1}$ increases to double the value of P $n$ BA (see Table 2 ), which goes parallel with an increase in $\Delta C_{\mathrm{p}} / x_{1}$. Both the value of $T_{2} \quad T_{1}$ and $\Delta C_{\mathrm{p}} / x_{1}$ are, thus, connected to the asymmetry caused by the small phase size. At the same time, the higher $T_{\mathrm{g}}$, attributed to the block $\mathbf{M} / \mathbf{A}$, shifts to lower temperatures with higher concentration of $n \mathrm{BA}$, as one would expect for such copolymers (see Tables 1 and 2). A comparison of $T_{\mathrm{g}}, T_{\mathrm{b}}$, and $T_{\mathrm{e}}$ of the gradient block copolymers with A $\mathbf{M} / \mathbf{A} 1$ reveals that this broadening is less asymmetric than the size effect, all three temperatures move to lower values, as seen best from Fig. 1(c) and (d). The gradient blocks in the copolymer with changing $n \mathrm{BA}$ concentration exhibit, thus, a broadening in the glass transition due to the copolymerization, but there is no additional phase separation within the M/A-block. The samples of $\mathbf{A} / \mathbf{M} \quad \mathbf{A} \quad \mathbf{M} / \mathbf{A} \mathbf{2}$ to $\mathbf{A} / \mathbf{M} \quad \mathbf{A}$ M/A4 of Fig. 2 are again, as pointed out above, showing an almost constant behavior of the lower glass transition. With higher $n \mathrm{BA}$ content in the M/A-blocks, it gets increasingly difficult to establish the now overlapping glass transitions.

\section{Conclusions}

This calorimetric analysis has shown for the first time that it is possible to separate size and solubility effects for block copolymers. The size-effect leads to an asymmetric 
broadening of the glass transition. The solubility (copolymerization) leads to a shift in the glass transition, and, as long as there are homopolymeric sections in the copolymer, a symmetric broadening of the transition is superimposed. Furthermore, these first measurements allow the speculation that with higher precision in the calorimetry, as is possible by using multi-frequency temperature-modulated DSC [22], a more detailed description of the phase-contours within the samples may be possible. Of particular interest would be the analysis of $n \mathrm{BA} / \mathrm{MMA}$ gradient copolymers which have sufficiently long Pn BA and PMMA ends to be able to produce a layer structure with a diffuse interphase.

\section{Acknowledgments}

This work was supported by the Division of Materials Research, National Science Foundation, Polymers Program, Grant Nos. DMR-9703692 and DMR-0090409 and the Division of Materials Sciences and Engineering, Office of Basic Energy Sciences, US Department of Energy at Oak Ridge National Laboratory, managed and operated by UTBattelle, LLC, for the US Department of Energy, under contract number DOE-AC05-00OR22725.

\section{References}

[1] Chen W, Wunderlich B. Nanophase separation of small and large molecules, invited feature article. Macromol Chem Phys 1999;200:283.
[2] Matsen MW, Bates FS. J Chem Phys 1997;106:2436.

[3] Zielinski RP, Childers CW. Rubber Chem Technol 1968;41:161.

[4] Kraus G, Rollman KW. Angew Makromol Chem 1971;16/17:271.

[5] Hashimoto T, Tsukahara Y, Tachi Y, Kawai H. Macromolecules 1983;16:648.

[6] Legge NR, Holden G, Schroeder HH, editors. Thermoplastic elastomers. Munich: Hanser; 1987.

[7] Greszta D, Matyjaszewski K, Pakula T. Polym Prepr ACS 1996;37(1): 569.

[8] Pakula T, Matyjaszewski K. Macromol Theory Simul 1996;5:987.

[9] Di Lorenzo ML, Pyda M, Wunderlich B. J Polym Sci, Part B: Polym Phys 2001;39:1594.

[10] Di Lorenzo ML, Pyda M, Wunderlich B. J Polym Sci, Part B: Polym Phys 2001;39:2969.

[11] Matyjaszewski K, Greszta D, Pakula T. Polym Prepr ACS 1997;38(1): 707.

[12] Matyjaszewski K, Ziegler M, Arehart SV, Greszta D, Pakula T. J Phys Org Chem 2000;13:775.

[13] Wang J S, Matyjaszewski K. J Am Chem Soc 1995;117:5614.

[14] Matyjaszewski K, Xia J. Chem Rev (Washington DC) 2001;101:2921.

[15] Matyjaszewski K, Shipp DA, McMurray GP, Gaynor SG, Pakula T. J Polym Sci, Part A: Polym Chem 2000;38:2023.

[16] Wunderlich B. Heat capacity of polymers. In: Cheng SZD, editor. Handbook of thermal analysis and calorimetry, vol. 3. Amsterdam: Elsevier; 2002.

[17] Archer DG. J Phys Chem Ref Data 1993;22:1441.

[18] Pyda M, editor. Advanced thermal analysis system (ATHAS). DATA Bank, http://web.utk.edu/ athas.

[19] Gaur U, Wunderlich B. Macromolecules 1980;13:1618.

[20] Bopp RC, Gaur U, Kambour RP, Wunderlich B. J Therm Anal 1982; 25:243.

[21] Lau SF, Pathak J, Wunderlich B. Macromolecules 1982;15:1278.

[22] Kwon YK, Androsch R, Pyda M, Wunderlich B. Thermochim Acta 2001;367/368:203 